\title{
Surface forces between rough and topographically structured interfaces
}

\author{
Thormann, Esben
}

Published in:

Current Opinion in Colloid \& Interface Science

Link to article, DOI:

10.1016/j.cocis.2016.09.011

Publication date:

2017

Document Version

Peer reviewed version

Link back to DTU Orbit

Citation $(A P A)$ :

Thormann, E. (2017). Surface forces between rough and topographically structured interfaces. Current Opinion in Colloid \& Interface Science, 27, 18-24. https://doi.org/10.1016/j.cocis.2016.09.011

\section{General rights}

Copyright and moral rights for the publications made accessible in the public portal are retained by the authors and/or other copyright owners and it is a condition of accessing publications that users recognise and abide by the legal requirements associated with these rights.

- Users may download and print one copy of any publication from the public portal for the purpose of private study or research.

- You may not further distribute the material or use it for any profit-making activity or commercial gain

- You may freely distribute the URL identifying the publication in the public portal

If you believe that this document breaches copyright please contact us providing details, and we will remove access to the work immediately and investigate your claim. 


\title{
Surface forces between rough and topographically structured interfaces
}

Esben Thormann, Technical University of Denmark, Department of Chemistry, DK-2800 Kgs. Lyngby, Denmark.

\begin{abstract}
Within colloidal science, direct or indirect measurements of surface forces represent an important tool for developing a fundamental understanding of colloidal systems, as well as for predictions of the stability of colloidal suspensions. While the general understanding of colloidal interactions has developed significantly since the formulation of the DLVO theory, many problems still remain to be solved. One concrete problem is that the current theory has been developed for interaction between flat and chemically homogenous surfaces, which is in contrast to the surfaces of most natural and manufactured materials, which possess topographical variations. This property is commonly referred to as surface roughness. Further, with technological advances in nanotechnology, fabrication of nano- or micro-structured surfaces has become increasingly important for many applications, which calls for a better understanding of the effect of surface topography on the interaction between interfaces. This paper presents a review of the current state of understanding of the effect of surface roughness on DLVO forces, as well as on the interactions between topographically structured hydrophobic surfaces in water. While the first case is a natural choice because it represents the most general description of colloidal interactions, the second case represents examples of how intentionally built-in surface structures can significantly alter the interactions between surfaces.
\end{abstract}

\section{Introduction}

The variation in surface topography, often referred to as surface roughness, is a natural part of all surfaces, with a few exceptions. Together with the media in which a surface is located, the bulk properties of the material and the surface chemistry, the surface topography is a key parameter in understanding surfacerelated phenomena, such as wetting, adhesion, adsorption, and friction. While the correlation between topography and wetting has been a hot topic in past decades, there is still little understanding of the effect of surface topography on the remaining topics. Surface topography represents a challenge in studies of interactions between interfaces, which is often experimentally avoided by the use of flat, model surfaces such as mica or silicon wafers. However, for the surfaces of natural or fabricated materials such as steel, ${ }^{1}$ calcium carbonate, ${ }^{2}$ and silicon oxide, ${ }^{3}$ or for deposited layers of, for example, gold ${ }^{4}$ titanium $^{5}$ and polymers, ${ }^{6}$ surface topography cannot be neglected without significant discrepancies between theory and experimental results. In other cases, such as for nanostructured devices ${ }^{7}$ and nanopatterned surfaces, ${ }^{8}$ surface topography is a feature which is intentionally built-in, in order to produce or enhance certain functional properties, such as specific adhesion, ${ }^{9}$ controlled reflectivity, ${ }^{10}$ self-cleaning ${ }^{11}$ and directional controlled wettability ${ }^{12}$. 
A lack of understanding of the effect of surface topography is problematic because it prevents theoretical predictions of the conditions for colloidal stability, and hampers the interpretation of experimentally obtained force-distance data. It can further lead to direct misinterpretations of experimental deviations from theory, which are often explained by the influence of surface roughness without any further proof. On the other hand, a full understanding of the influence of surface topography can potentially offer a new additional tool for "tuning" the interaction between two surfaces by a particular design of the surface topography. This could, for example, be used to enhance or reduce colloidal stability in line with the use of sterically repulsive polymer layers and regulation of electrostatic interactions by variations in salt concentration or $\mathrm{pH}$.

Although the effect of surface topography on surface forces is not substantially implemented in the surface force theories, it is a field of growing interest and development. Thus far, various studies of the effect of surface topography on capillary force, ${ }^{13-15}$ surface contact mechanics, ${ }^{16-18}$ Casimir forces, ${ }^{19-21}$ hydration forces, ${ }^{22}$ and steric forces ${ }^{23}$ have been conducted. This short review is limited to the discussion of the effect of roughness on DLVO forces, i.e., the electrical double layer force and van der Waals forces, and the effect of nanometre-to-micrometre topographical surface variation on the interaction between hydrophobic surfaces in water.

\section{DLVO forces}

The DLVO theory represents the classical description of interactions between colloids in a liquid medium and consists of the sum of short-ranged van der Waals forces and long-ranged electrical double layer forces. ${ }^{24,} 25$ It is well-known that this theory builds on certain assumptions and has limitations due to, for example, ion-ion correlations and specific ion hydration effects. ${ }^{26-29}$ However, in the context of this review, an important assumption is that the interactions are described to occur between perfectly smooth and chemically homogeneous surfaces. With respect to electrical double layer forces, it implies that the double layer potential is smeared out and that surface potentials and charge densities of two interacting surfaces can be described by a single variable, i.e., the distance between the surfaces. ${ }^{30}$ For van der Waals forces, the assumption implies that the interaction potential is characterized by a single adjustable parameter, the Hamaker constant, which does not vary with distance as long as retardation effects are neglected. For both cases, these assumptions are obviously challenged when surface roughness is introduced.

Although the effect of roughness is often ignored in the evaluation of experimentally obtained surface force data, several theoretical ${ }^{17,30-35}$ and experimental ${ }^{17,}$ 33, 35-38 studies have attempted to investigate its effect on DLVO forces in a more or less systematic way. However, before going into the findings and methods of dealing with surface roughness, it is appropriate to address an apparent disagreement in literature about whether DLVO forces are generally enhanced ${ }^{2-4,8}, 10,11$ or reduced $^{33-35,39,40}$ by surface roughness. This is not a real disagreement but rather a matter of different definitions of the separation distance between two rough interfaces. In the former works, the separation distance, $D_{m p}$, is defined as the distance between the midplanes of the surface asperities, while in the latter works, the separation distance, $D_{\min }$, is defined as the distance between the first points of mechanical contact (see Figure 1a). Thus, if using the former definition, the zero-separation distance is hidden behind the point of mechanical 
contact and the surfaces cannot be brought closer than the peak-to-valley distance before repulsive contact forces kick in. While this definition has some computational advantages, it is, in the authors opinion, a bit misleading because it is in contrast to the experimental observations, in which the effective DLVO forces before mechanical contact and elastic (or hard-wall) repulsion are reduced by surface roughness ${ }^{41}$ - e.g., aggregation between rough particles due to attractive van der Waals forces is less likely than between smooth particles of the same material ${ }^{38,42}$.

Basically, the effect of roughness on the DLVO forces has been treated in three different ways. In the first type of approach, the surface roughness has either been modelled by a certain geometry and density of surface asperities $^{31,32,34,36}$, by a probability distribution ${ }^{17,35}$, by a lattice model ${ }^{30}$ or directly measured by AFM imaging ${ }^{17,37}$, and the interaction potentials have subsequently been obtained by appropriate mathematical techniques, such as surface element integration techniques or a mean-field lattice analysis. These studies demonstrate good agreement between theory and experimental results - especially when a repulsive contribution for elastic deformation of the surface asperities is included. It is demonstrated that the effect of roughness becomes pronounced when the separation distance between the surfaces becomes comparable with the magnitude of the amplitude roughness parameters. For typical surfaces with an amplitude roughness on the order of 1-10 nm, it will strongly affect van der Waals forces, which also typically become significant in this interaction range. By defining the zero-separation distance at the midplane of the amplitude roughness, $D_{m p}$, the surface roughness is shown to amplify the van der Waals forces independently of the surface roughness model and the integration method. However, as the plane of mechanical contact, $D_{\min }$, is shifted outwards as the amplitude roughness increases, the van der Waals forces at the point of first mechanical contact decrease with increasing roughness (see Figure 1). These models therefore support the general assumption that surface roughness suppresses the impact of van der Waals forces in surface force measurements (see Figure 1c). Similarly, electrical double layer forces are enhanced or reduced based on the definition of zero-separation. However, because the roughness only plays a significant effect at small surface separations, the effect on electric double layer forces is weak at low salt concentrations, i.e., when the length scale of the amplitude roughness is much smaller than the Debye length. Oppositely, at high salt concentrations (or for extremely rough surfaces), surface roughness will also significantly affect the electrical double layer force. The effects of different salt concentrations and different roughness scales on the electrical double layer force are illustrated in Figure $1 \mathrm{~b}$. In conclusion, this approach can be considered as a very exact method for incorporating the effect of surface roughness on DLVO forces. It explains both the qualitative effects and can, to some extent, produce an explicit functional form of the interaction potential, which is in good agreement with experimental results. However, to achieve good quantitative agreement between theory and experimental results, very precise knowledge of the topography of the interacting surfaces is required and such information is not always practically easy to obtain. Another drawback of the approach is the increased mathematical complexity compared to the "standard DLVO theory", which might limit its use as a general approach.

In the second type of approach, the rough interface is modelled as either a homogenous or a diffuse layer of a thickness corresponding to the length-scale of the amplitude roughness and with values for the dielectric properties in between the values of the bulk material and the solvent. ${ }^{33,43}$ By this approach, van der Waals forces between rough surfaces can easily be modelled by a single layer or a multilayer approach, as originally suggested by Ninham and Parsegian for thin films on two half spaces. ${ }^{4-46}$ The single layer model can qualitatively reproduce the weakening of the van der Waals forces due to surface roughness. As 
one can draw direct parallels to the effect of thin polymer coatings or supported lipid bilayers, it is also very intuitive for the understanding of why roughness has a strong influence at short separation distances and a vanishing effect at large separations. However, as the volume fraction of the bulk material is not homogenous but normally a decreasing function of the distance from the surface, a multilayer or diffuse layer model is required to also quantitatively reproduce the functional form of the interaction potential. Thus, in order to obtain quantitative agreement between theory and experiments, precise knowledge of the surface topography of the interacting surfaces is needed. Further, the suggested approach is only applicable to van der Waals forces and not to electrical double layer forces, which means that the approach is sufficient only at low salt concentrations, where the effect of surface roughness primarily affects the van der Waals forces (short-ranged forces), and affects the electrical double layer force (long-range force) only to a minor extent.

The third type of approach is a pragmatic but non-exact approach to account for the apparent roughnessinduced reduction in the van der Waals forces. ${ }^{37,38,40,47,48}$ Although the routes and arguments are different in the different works, the end result is basically that the plane of zero-separation is shifted inwards. This manoeuvre thus effectively eliminates the most attractive part of the van der Waals interaction, which is not manifested due to hard-wall or elastic-contact repulsive forces. Therefore, this brings experimentally obtained force-distance data, with an apparent lack of short-range attraction, in qualitative agreement with the DLVO theory, but without reproducing the exact functional form of the interaction potential. However, at low salt concentrations, an exact functional form of the van der Waals forces is often of minor importance as long as the amplitude length scale of the surface roughness is much smaller than the Debye length.

We examined three different approaches to account for the effect of surface roughness on the DLVO forces. These three approaches all confirm that surface roughness suppresses the effect of short range DLVO forces, as observed from the point of first mechanical contact. This conclusion is important because it confirms the often used explanation that surface roughness is responsible for the apparent lack of strong short-ranged attractive van der Waals forces in experimentally obtained force-distance data (see illustration in Figure 1c). The best quantitative results are obtained for surfaces with very specific surface geometries or if experimentally obtained surface roughness profiles are applied. While such results are important in order to confirm the validity of the approach, it is, in the author's opinion, less applicable as a general method. The DLVO theory is already a generalized theory that neglects, for example, ion correlation effects and ion specificity. Further, for practical use, the electrical double layer force always gives a poor description of the interactions at short separations, due to the use of fixed boundary conditions such as constant charge or constant potential. Thus, it makes little sense to add specific and difficult-to-achieve requirements in order to obtain a very exact description on the effect of roughness at short separations, to a model that is generically unprecise at such short separations. Instead, a generalized description of the roughness profile, which captures the essential features, is more appropriate for a generalized theory. In a recent study by Parsons et al., ${ }^{17}$ experimental results of the interaction between titanium surfaces with a RMS roughness of $9.6 \mathrm{~nm}$ are compared to a roughness model where the roughness profile is assumed to be Gaussian (with a standard variation corresponding to the RMS roughness) and measured by AFM imaging, respectively. In this concrete example, the two approaches yield very similar results, down to separations of approximately $10 \mathrm{~nm}$, i.e., to a distance approximately corresponding to the value of the RMS roughness. However, more studies on surfaces with different roughness profiles are needed in order 
to determine if the Gaussian roughness profile can work as a general approach and to find the limitations in terms of surface separation range compared to the roughness scale.

\section{Forces between hydrophobic surfaces}

In the case of DLVO forces, surface topography is an issue that needs to be addressed due to the intrinsic roughness of the surfaces of most natural and manufactured materials. For hydrophobic surfaces, surface topography is not only relevant because of the effects of intrinsic roughness but also because surface topography, in many cases, is a part of an intentional design of the surfaces. Superhydrophobic surfaces are defined as surfaces with water contact angles higher than $150^{\circ}$ and have, during the last two decades, attracted increasing interest due to their highly water-repellent, self-cleaning, anti-icing, and drag-reducing properties. ${ }^{49-53}$ It is well-known that such high contact angles can only be achieved by a combination of surface chemistry and surface topography. The surface topography, which is originally inspired by biointerfaces such as lotus leaves, rose petals, and the legs of water striders, is composed of hierarchical structures ranging from the nanometre scale to the micrometre scale (see Figure 2). ${ }^{50,54-57}$ Two principle models, the Cassie-Baxter mode ${ }^{58}$ and the Wenzel mode ${ }^{59}$, are commonly used to describe the wetting on such structured surfaces, although many real cases show a combination of the two situations. As shown in Figure 2, the lotus leaf is a typical example of a surface where the wetting behaviour follows the CassieBaxter model. Here, the aqueous phase partially wets the surface features but is also resting on air trapped in the surface depressions. The surface of the rose petal constitutes an example of a more open surface structure where the aqueous phase penetrates the microstructures. As discussed later, this difference might be very important for the interaction between such surfaces. However, while the wetting behaviour of superhydrophobic and other topographical structured surfaces has been extensively studied and is relatively well-described by the Wenzel and Cassie-Baxter states, the interactions between such surfaces in an aqueous medium have been investigated to a much smaller extent. A reason for this is probably that even the interaction between two flat hydrophobic surfaces in water is still not fully understood and the further complication of surface topography has thus been avoided.

The attractive interaction between flat hydrophobic surfaces in water has been a controversial topic since the first direct measurements by Israelachvili and coworkers in 1982, and the suggestions for the origin of this attraction are numerous. ${ }^{60-65}$ However, although there is still an ongoing debate about this topic, it seems that cavitation or bridging nanobubbles can explain many of the phenomena reported in literature. ${ }^{66-70} \mathrm{~A}$ more thorough discussion about the origin of hydrophobic interactions is, however, not in the scope of this paper and the reader is referred to various reviews on the topic. ${ }^{63,}{ }^{71-73}$ Instead, this discussion focuses on how the attractive interaction, caused by the formation of a bridging air/vapour cavity, is affected by surface topography. Before moving on to the interaction between topographically structured surfaces, the profile of a typical force-distance curve between two flat hydrophobic surfaces will be briefly discussed. Figure 3a shows a typical force curve obtained by the AFM colloidal probe technique, between a flat silica surface and a silica microsphere, both functionalized by a hydrophobic silane. On approach, no detectable force is observed before a certain separation distance (here $16 \mathrm{~nm}$ ), at which a sudden attraction appears and the surfaces are brought into contact in discontinuous jumps. This sudden attraction is assigned to the formation of an air/vapour cavity. Upon retraction, a long-ranged diminishing 
attraction, corresponding to the elongation of the bridging cavity, persists until a certain separation (here $63 \mathrm{~nm}$ ), at which the cavity ruptures and the attraction disappears. This shape of the retraction force curve has an important fingerprint of the force law for elongation of a capillary bridge with a constant volume. The force law, which is originally derived for a water capillary bridging two hydrophilic surfaces in a humid atmosphere, but which also works for a bridging air bubble between two hydrophobic surfaces in water, is given as: ${ }^{74}$

$F=4 \pi \gamma c R\left(1-\frac{D}{\sqrt{\frac{V}{\pi R}+D^{2}}}\right)$

where

$c=\frac{\cos \left(\theta_{1}+\beta\right)+\cos \theta_{2}}{2}$

and $\gamma$ is the surface tension of water, $D$ is the separation distance, $V$ is the capillary volume, $R$ is the radius of the colloidal probe, $\theta_{1}$ and $\theta_{2}$ are the contact angles of the capillary against the each of the surfaces, and $\beta$ is a geometrical parameter that can be ignored for hydrophobic surfaces where $\beta<<\theta$. The strong agreement between this theoretical force law and experimental observations strongly suggest that no extra air is supplied to the cavity after its formation and subsequent elongation.

Although the number of studies of interactions between superhydrophobic or other topographically structured surfaces is small compared to the number of studies of the interactions between flat hydrophobic surfaces, some important effects of the surface topography have been observed. The first reported study of the interactions between superhydrophobic surfaces, by Singh et al., employed interfacial force microscopy, showed a much more long-ranged attraction than observed for flat hydrophobic surfaces. $^{75}$ Later, Brandner et al. and Wåhlander et al. reported more detailed force-distance data for interactions between two superhydrophobic surfaces, obtained by the AFM colloidal probe technique. ${ }^{76,77}$ As exemplified in Figure $3 b$, these studies showed a force-distance profile which clearly does not follow the force law given by equation 1 . In line with other previous studies of the interactions between topographically structured surfaces, ${ }^{78-80}$ the authors suggest that the deviation from equation 1 is a result of continuous supply of air from the (dewetted) surface features to the bridging cavity during elongation. Consequently, the correlation between the nature of the superhydrophobic surfaces and "the new type" of bubble-induced forces between such surfaces seems obvious. The high contact angles of a water droplet on superhydrophobic surfaces are, in this case, caused by Cassie-Baxter wetting (see Figure $2 \mathrm{c}$ and $3 \mathrm{~b}$ ), which indicates that the surface region is composed of a reservoir or air that can be supplied to the bridging cavity as it is elongated. Although the exact appearance of the force-distance profile varies between repeated measurements and for different surfaces, it is interestingly observed that the air seems to be supplied at a rate which gives rise to a constant or weakly increasing force during the elongation of the cavity. This indicates that the driving force for the air supply is the surface tension of the bridging cavity and that the rate at which air is supplied is (at least partly) regulated in order to maintain a constant surface-to-volume ratio in the cavity. However, in order to conclude if this is a universal behaviour, more studies with systematic variations in contact time and retraction speed are required. 
While the qualitative effect of topographical structures on the interaction between hydrophobic surfaces in water shows that increased accessibility of air at the interface region can increase the size of the bridging cavity and result in a non-constant volume of the cavity upon elongation, quantification of the effect is challenging. This is because meaningful parametrization of the surface topography of superhydrophobic surfaces is difficult in most cases. In other cases, a comparison between different studies requires a comparison of the interactions between surfaces with different surface chemistry or with different macroscopic contact angles, which makes it difficult to separate the effect of different topographies from other properties. However, in one study, Hansson et al. ${ }^{78}$ have managed to separate these parameters by preparing a series of topographically structured hydrophobic surfaces with a systematic variation in both the amplitude and spatial roughness parameters, but with the same surface chemistry and macroscopic contact angles. These surfaces were built up by monolayers of hexagonally closed-packed sintered and silanized silica particles of sizes ranging from 30 to $800 \mathrm{~nm}$ and $R_{q}$ roughness values ranging from approximately 5 to $50 \mathrm{~nm}$ (see Figure 4). All of these surfaces all displayed water contact angles of approximately $120^{\circ}$, which was shown to correspond to complete wetting of the top hemispheres of the particles and presumably, a continuous air layer surrounding the bottom hemispheres resting on the solid support. ${ }^{81}$ Hereafter, the interaction between each of these surfaces and a micrometre sized silanized silica particle were measured in water by the AFM colloidal probe technique. It was found that for each sample, a given fraction of the force curves displayed behaviour similar to the interaction between flat hydrophobic surfaces (bridging cavity with a constant volume) while another fraction displayed behaviour similar to the interaction between superhydrophobic surfaces (bridging cavity with a growing volume). Interestingly, it was found that the second type of behaviour was observed very frequently for interactions between the probe and the surfaces with smaller particles, and very seldom for the surfaces with larger particles. Since all surfaces were expected to house a reservoir of air within the surface features, this difference was explained by the length-scale effect on the cavity formation and growth. Further, when accounting for the volume of the crevices between the particles, the formation of a bridging cavity required a much larger total volume in the case of the larger particles than in the case of the smaller particles. It was further speculated that possible pinning of the three-phase line could hamper the capillary growth in the case of larger particles. In summary, this means that the surface structure provides an air reservoir that promotes capillary growth while an excessively large magnitude of surface features has the opposite effect. Therefore, the optimal structures to promote stronger capillary interactions between hydrophobic surfaces seem to be nanostructures rather than microstructures. This also illustrates that simple roughness parameters, such as the RMS roughness, appear to provide a poor description of the topographical effects because it is the actual morphology on the nanometre scale that enables capillary growth by the inflow and accessibility of air, while the long-range micrometre scaled structure both determine the size of the air reservoir and can promote pinning effects.

\section{Conclusions and Outlook}

This review discusses the effect of surface topography on the forces between surfaces in water and, more specifically, on DLVO forces and capillary interactions between hydrophobic surfaces. DLVO forces exist between all surfaces in water and are the cornerstone of theories for surface forces. They are thus a natural starting point for a discussion about the effect of surface topography, in order to obtain agreement 
between theory and experiment. Conversely, the interaction between hydrophobic surfaces in water constitutes a very specific case but here, surface topography has a well-documented effect on the properties of hydrophobic surfaces, which thus represent powerful examples of the potential for manipulation of surface properties by understanding and actively using surface topography in the surface design.

In the case of DLVO forces, it has been shown that the effect of surface roughness leads to a reduction of both attractive van der Waals forces and repulsive electrical double layer forces, as observed from the point of first mechanical contact. However, because the roughness has the largest effect for short separation distances, the two contributions to the total DLVO force are not the same. While the van der Waals forces are always significantly reduced, the electrical double layer force is mostly affected at high salt concentrations, i.e., when the magnitude of the Debye length becomes comparable with the magnitude of the amplitude roughness. This effect of the surface roughness thus explains many experimental results of interactions between two rough surfaces, which seem to be dominated by repulsive electrical double layer forces, while the short-ranged attractive van der Waals force appears to be missing. Despite the success of the explanation of "lacking" van der Waals forces, a simple and generalized formalism for the effect of roughness still needs to be verified. Thus far, most studies have adopted a formulation that requires detailed knowledge about the surface topography, which is in contrast to the very general and simplistic approach of classical DLVO theory. Currently, the best candidate for a generalized theory appears to be an approach where the surface roughness variations are treated as a Gaussian distribution.

The primary difference between the interactions between flat hydrophobic surfaces and those between topographically structured surfaces is more difficult to quantify. However, it can be summarized by the following key points: (1) in many cases, the interactions between hydrophobic surfaces in water can be described by cavitation and formation of an air/vapour capillary bridge between the surfaces. (2) In the case of flat hydrophobic surfaces, the force-distance curve upon separation fits the model of elongation of a capillary bridge of constant volume. (3) On rough or topographically structured surfaces, a deviation from the constant-volume behaviour is often observed. (4) The deviation from the constant-volume behaviour is more pronounced for nanostructured surface features than for micro-structured surface features. All these observations suggest that nano-scaled surface features can work as air reservoirs, which can diffuse into the bridging cavity during cavity formation and/or elongation, thereby leading to stronger attractive interactions. Finally, it should be noted that the cases discussed in this review involve surfaces where air is trapped within the surface structures. However because this is not the case for all topographically structured hydrophobic surfaces, a direct comparison of interactions between Cassie-Baxter wetted and Wenzel wetted surfaces could possibly help to further shine light on this topic.

Future perspectives for studies related to the effect of surface topography and the interactions between surfaces or between colloids can be divided in two parts. One part, which has already been discussed above, regards the future development of theories in order to treat the effects of topography in a simple and generalized way. The second part involves new potential applications of this knowledge. In previous works on enhancing the colloidal stability of various suspensions, electrostatic, steric, and electro-steric forces have received attention for counteracting the attractive van der Waals forces. However, current knowledge of the effect of surface roughness on DLVO forces indicates that it can also be used actively to 
control and enhance colloidal stability by effectively decreasing the attractive van der Waals forces with increased roughness.

\section{Acknowledgment}

The Swedish research council (VR) is acknowledged for financial support by a research grant which has been transferred to the Technical University of Denmark via the European initiative "Money Follows Researcher". Anne Frejberg is acknowledged for help with the illustrations in Figure 2 and 3.
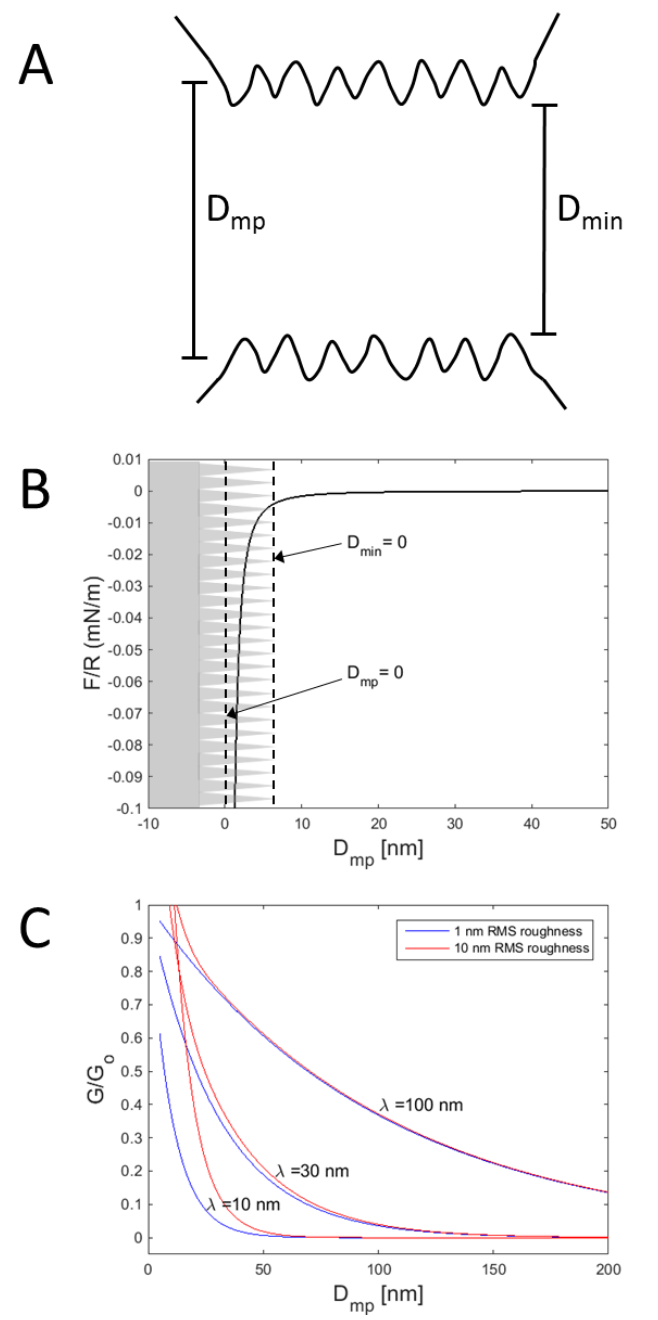

Figure 1: (A) Because the separation distance between two topographically structured surfaces is not uniquely defined, DLVO forces can be considered to be either enhanced or reduced by the surface structure. The two most common definitions of the separation distance are the distance between the weighted midplanes of the surface asperities, $D_{m p}$, and the distance between the outer parts of the surface asperities, $D_{\min }$. The latter definition thus sets the point of zero separation as the first point of mechanical contact, while by the former definition, the point of zero separation cannot be reached due to elastic or 
hard-wall repulsion. (B) Calculated van der Waals forces between a smooth spherical particle and a plane surface with $10 \mathrm{~nm}$ asperities. As observed here, the attractive interaction is weak at the point of first mechanical contact, $D_{\min }=0$, explaining the apparent lack of strong attraction observed in force-distance data for interactions between rough surfaces. A non-retarded Hamaker constant of $A=10^{-21} \mathrm{~J}$ was used for this calculation. (C) Normalized electrical double layer energies for interactions between two sets of identical surfaces with RMS roughness of 1 and $10 \mathrm{~nm}$, respectively, and for three different Debye lengths. $G_{0}$ is the prefactor for the interaction potential, which depends of the surface charge and surface potential. A significant effect of surface roughness is observed for small Debye lengths (high salt concentrations) while the interaction energy is almost unaffected for larger Debye lengths (low salt concentrations). For more details about these calculations, the reader is referred to the supporting information of the work by

Parsons et al. ${ }^{17}$
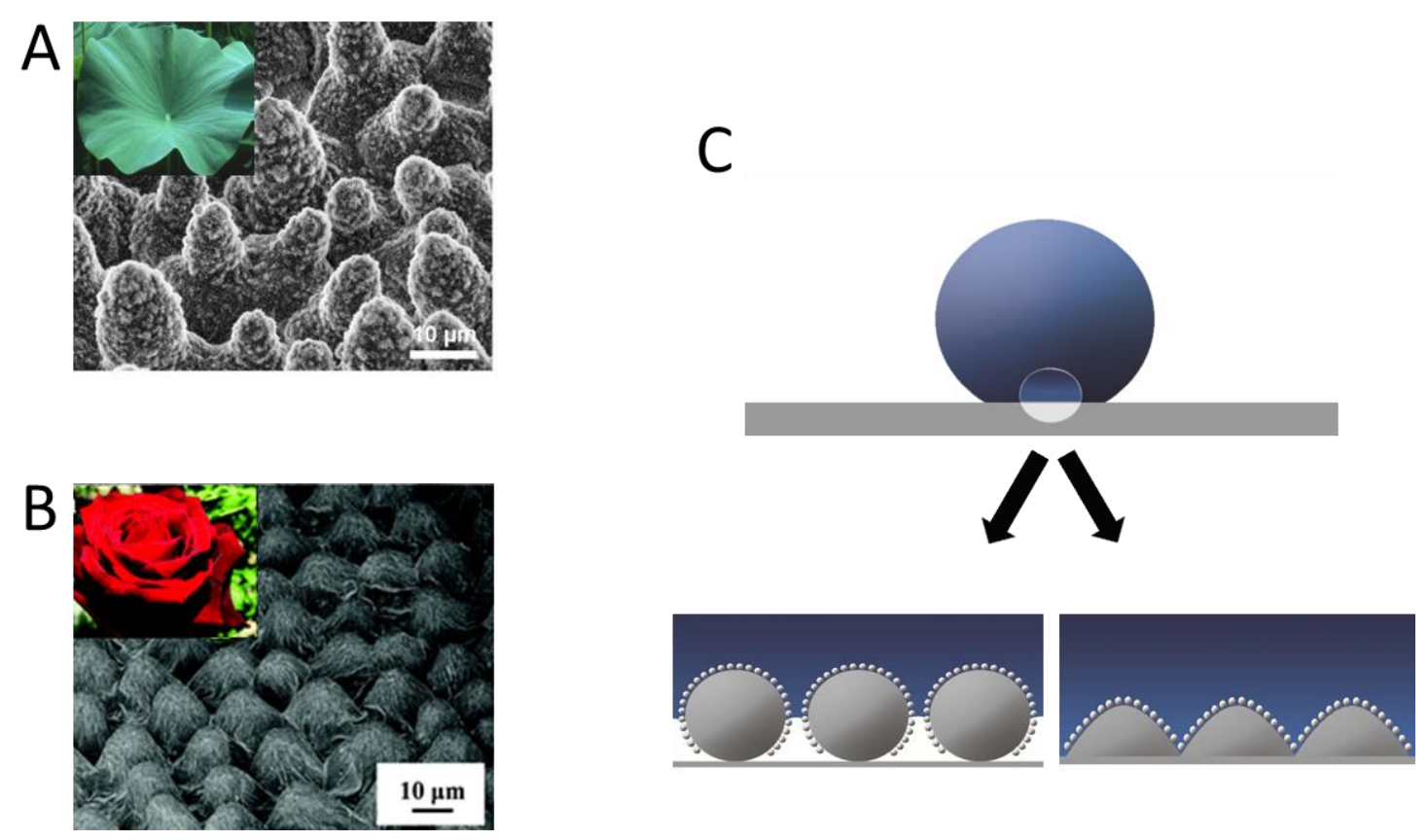

Figure 2: (A) SEM image of a lotus leaf, showing the hierarchical surface topography with variations from the nanometre scale to the micrometre scale (the figure is reproduced from Ensikat et al. ${ }^{82}$ (open access source)). (B) SEM image of a rose petal showing the more open hierarchical surface topography compared to the lotus leaf (the figure is reproduced from Feng et al. ${ }^{56}$ with permission from the American Chemical Society). (C) Illustration of the two wetting states, commonly known as the Cassie-Baxter and the Wenzel states, which are characterized by partial and complete wetting, respectively. 

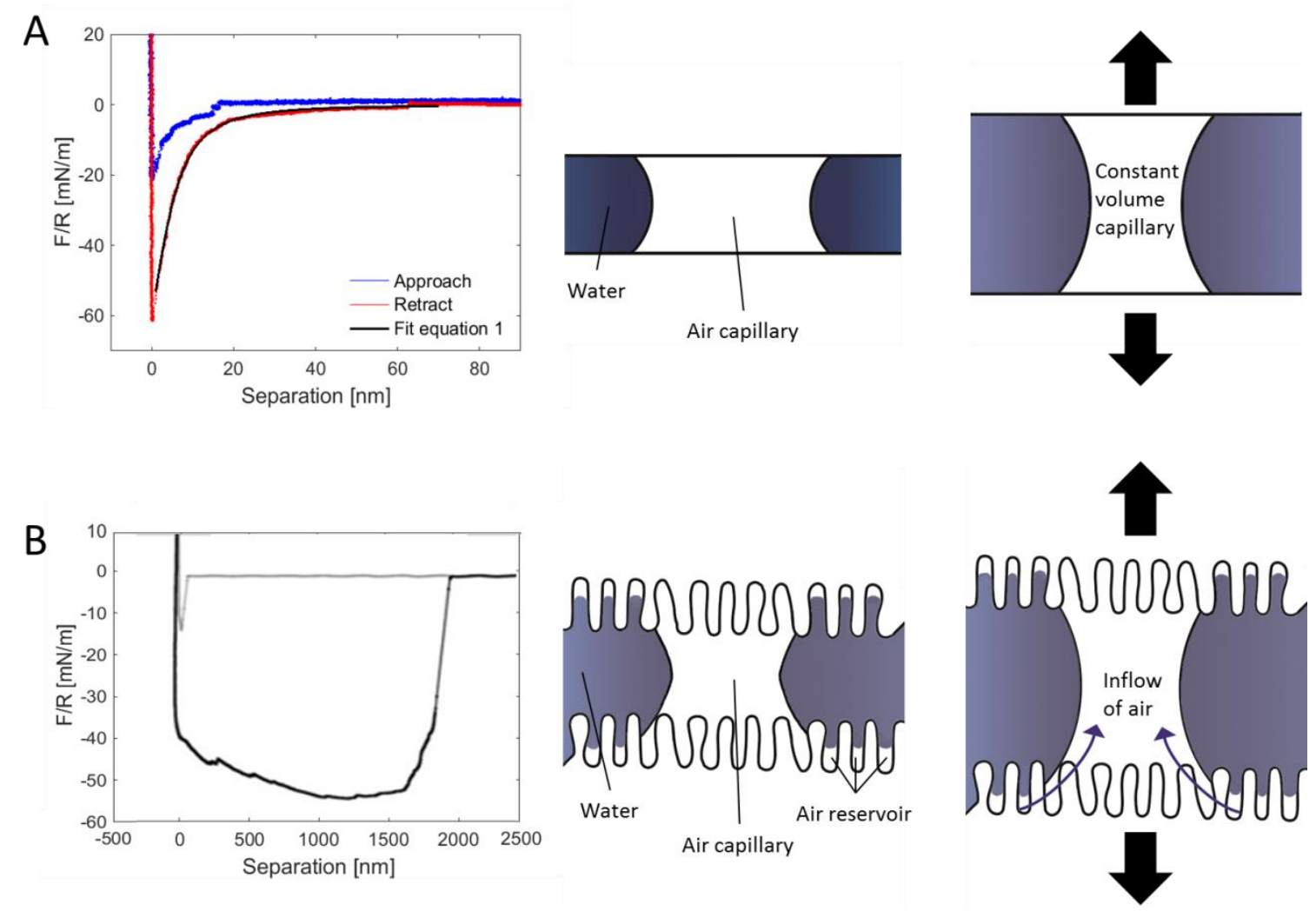

Figure 3: (A) Interaction between a flat hydrophobized silica surface and a hydrophobized silica colloidal probe in water. Upon approach, a sudden attraction is observed at a separation of $16 \mathrm{~nm}$, which is attributed to the formation of a bridging air/vapor cavity. Upon retraction, a long-ranged attraction, which is attributed to the elongation of the bridging cavity, is observed until a separation of $63 \mathrm{~nm}$, at which point the cavity ruptures. The black dashed line represents the fit of the model of elongation of a bridging cavity with a constant volume (equation 1). The details of this experiment have been previously described by Thormann et al. ${ }^{69}$ and the figure is reproduced with permission from the American Chemical Society. (B) Interaction between two superhydrophobic surfaces in water. Upon approach, a sudden attraction is observed at approximately $60 \mathrm{~nm}$ that, as in the case of the flat surfaces, is assigned to the formation of a bridging air/vapor cavity. Upon retraction, a long-ranged attraction, which does not fit the model of elongation of a bridging cavity with a constant volume, is observed. This observation is attributed to a constant inflow of air from the reservoir of trapped air in the structures of the superhydrophobic surfaces, which leads to a non-constant volume of the cavity during elongation. The details of this experiment have been previously described by Wåhlander et al. ${ }^{77}$ and the figure has been reproduced with permission from 
Elsevier. The left part of the figure illustrates the difference between elongating a bridging air/vapor cavity between flat hydrophobic surfaces (constant volume) and between superhydrophobic surfaces (growing volume).

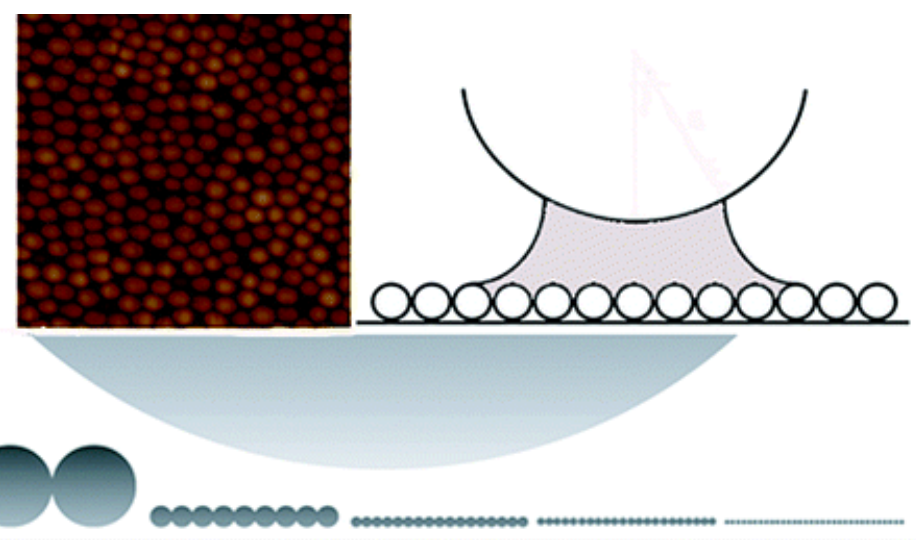

Figure 4: Schematic illustration of a surface design where both the lateral and amplitude roughness parameters are systematically changed. Because the wetted part of the surface is made up of hexagonally closed-packed hemispheres, the roughness factor and thus, the macroscopic contact angle becomes independent of the particle size and consequently, of the actual surface roughness.

\section{References and recommended reading, ${ }^{* * *}$}

* Of special interest.

** Of outstanding interest.

1.

Hilbert, L. R.; Bagge-Ravn, D.; Kold, J.; Gram, L. Influence of surface roughness of stainless steel on microbial adhesion and corrosion resistance. International Biodeterioration \& Biodegradation 2003, 52, 175-185.

$2 . \quad$ de Leeuw, N. H.; Parker, S. C. Surface structure and morphology of calcium carbonate polymorphs calcite, aragonite, and vaterite: An atomistic approach. Journal of Physical Chemistry B 1998, 102, 2914-2922.

3. Jackle, J.; Kawasaki, K. Intrinsic roughness of glass surfaces. Journal of Physics-Condensed Matter 1995, 7, 4351-4358.

4. Semaltianos, N. G.; Wilson, E. G. Investigation of the surface morphology of thermally evapourated thin gold films on mica, glass, silicon and calcium fluoride substrates by scanning tunneling microscopy. Thin Solid Films 2000, 366, 111-116. 

composition of flat titanium thin films as a function of film thickness and evapouration rate. Applied Surface Science 2005, 250, 252-267.

$6 . \quad$ Liu, C.; Thormann, E.; Claesson, P. M.; Tyrode, E. Surface Grafted Chitosan Gels. Part I. Molecular Insight into the Formation of Chitosan and Poly(acrylic acid) Multi layers. Langmuir 2014, 30, 8866-8877.

7. Valsesia, A.; Colpo, P.; Silvan, M. M.; Meziani, T.; Ceccone, G.; Rossi, F. Fabrication of nanostructured polymeric surfaces for biosensing devices. Nano Letters 2004, 4, 1047-1050.

8. Pallandre, A.; Glinel, K.; Jonas, A. M.; Nysten, B. Binary nanopatterned surfaces prepared from silane monolayers. Nano Letters 2004, 4, 365-371.

9. Puckett, S. D.; Taylor, E.; Raimondo, T.; Webster, T. J. The relationship between the nanostructure of titanium surfaces and bacterial attachment. Biomaterials 2010, 31, 706-713.

10. Hadobas, K.; Kirsch, S.; Carl, A.; Acet, M.; Wassermann, E. F. Reflection properties of nanostructure-arrayed silicon surfaces. Nanotechnology 2000, 11, 161-164.

11. Blossey, R. Self-cleaning surfaces - virtual realities. Nature Materials 2003, 2, 301-306.

12. Xia, D.; Brueck, S. R. J. Strongly anisotropic wetting on one-dimensional nanopatterned surfaces. Nano Letters 2008, 8, 2819-2824.

13. van Zwol, P. J.; Palasantzas, G.; De Hosson, J. T. M. Influence of roughness on capillary forces between hydrophilic surfaces. Physical Review E 2008, 78.

14. Jones, R.; Pollock, H. M.; Cleaver, J. A. S.; Hodges, C. S. Adhesion forces between glass and silicon surfaces in air studied by AFM: Effects of relative humidity, particle size, roughness, and surface treatment. Langmuir 2002, 18, 8045-8055.

15. Butt, H.-J. Capillary forces: Influence of roughness and heterogeneity. Langmuir 2008, 24, 4715-4721.

16. Yang, C.; Persson, B. N. J.; Israelachvili, J.; Rosenberg, K. Contact mechanics with adhesion: Interfacial separation and contact area. Ep/ 2008, 84.

17.** Parsons, D. F.; Walsh, R. B.; Craig, V. S. J. Surface forces: Surface roughness in theory and experiment. Journal of Chemical Physics 2014, 140. [systematic comparison between theory and experiment for DLVO forces between rough surfaces]

18. Persson, B. N. J. Contact mechanics for randomly rough surfaces. Surface Science Reports 2006, 61, 201-227.

19. van Zwol, P. J.; Palasantzas, G.; De Hosson, J. T. M. Influence of random roughness on the Casimir force at small separations. Physical Review B 2008, 77.

20. Neto, P. A. M.; Lambrecht, A.; Reynaud, S. Roughness correction to the Casimir force: Beyond the proximity force approximation. Europhysics Letters 2005, 69, 924-930.

21. Munday, J. N.; Capasso, F.; Parsegian, V. A.; Bezrukov, S. M. Measurements of the CasimirLifshitz force in fluids: The effect of electrostatic forces and Debye screening. Physical Review A 2008, 78.

22. Valtiner, M.; Kristiansen, K.; Greene, G. W.; Israelachvili, J. N. Effect of Surface Roughness and Electrostatic Surface Potentials on Forces Between Dissimilar Surfaces in Aqueous Solution. Advanced Materials 2011, 23, 2294-+.

23. Stiernstedt, J.; Nordgren, N.; Wagberg, L.; Brumer, H., III; Gray, D. G.; Rutland, M. W. Friction and forces between cellulose model surfaces: A comparison. Journal of Colloid and Interface Science 2006, 303, 117-123.

24. Derjaguin, B. V.; Landau, L. Theory of the stability of strongly charged lyophobic sols and of the adhesion of strongly charged particles in solution of electrolytes. Acta Physicochim. URSS 1941, 14, 633662.

25. Verwey, E. J. W.; Overbeek, J. T. G. Theory of the stability of lyophobic colloids. The interaction of particles having an electric double layer. Elsevier: Amsterdam, 1948.

26. Israelachvili, J. N. Intermolecular \& Surface Forces. 2 ed.; Academic Press: London, 1991. 
27.

Ninham, B. W.; Yaminsky, V. Ion binding and ion specificity: The Hofmeister effect and Onsager and Lifshitz theories. Langmuir 1997, 13, 2097-2108.

28. Ninham, B. W. On progress in forces since the DLVO theory. Advances in Colloid and Interface Science 1999, 83, 1-17.

29. Parsons, D. F.; Bostroem, M.; Lo Nostro, P.; Ninham, B. W. Hofmeister effects: interplay of hydration, nonelectrostatic potentials, and ion size. Physical Chemistry Chemical Physics 2011, 13, 1235212367.

30. Duval, J. F. L.; Leermakers, F. A. M.; van Leeuwen, H. P. Electrostatic interactions between double layers: Influence of surface roughness, regulation, and chemical heterogeneities. Langmuir 2004, 20, 5052-5065.

31. Suresh, L.; Walz, J. Y. Effect of surface roughness on the interaction energy between a colloidal sphere and a flat plate. Journal of Colloid and Interface Science 1996, 183, 199-213.

32. Bhattacharjee, S.; Ko, C. H.; Elimelech, M. DLVO interaction between rough surfaces. Langmuir 1998, 14, 3365-3375.

33.** Dagastine, R. R.; Bevan, M.; White, L. R.; Prieve, D. C. Calculation of van der Waals forces with diffuse coatings: Applications to roughness and adsorbed polymers. Journal of Adhesion 2004, 80, 365394. [Interesting discussion about van der Waals forces between rough surface by diffuse layer approch] 34. Hoek, E. M. V.; Agarwal, G. K. Extended DLVO interactions between spherical particles and rough surfaces. Journal of Colloid and Interface Science 2006, 298, 50-58.

35. $\quad$ Elzbieciak-Wodka, M.; Popescu, M. N.; Ruiz-Cabello, F. J. M.; Trefalt, G.; Maroni, P.; Borkovec, M. Measurements of dispersion forces between colloidal latex particles with the atomic force microscope and comparison with Lifshitz theory. Journal of Chemical Physics 2014, 140.

36. Suresh, L.; Walz, J. Y. Direct measurement of the effect of surface roughness on the colloidal forces between a particle and flat plate. Journal of Colloid and Interface Science 1997, 196, 177-190.

37. Valtiner, M.; Banquy, X.; Kristiansen, K.; Greene, G. W.; Israelachvili, J. N. The Electrochemical Surface Forces Apparatus: The Effect of Surface Roughness, Electrostatic Surface Potentials, and Anodic Oxide Growth on Interaction Forces, and Friction between Dissimilar Surfaces in Aqueous Solutions. Langmuir 2012, 28, 13080-13093.

38. Considine, R. F.; Drummond, C. J. Surface roughness and surface force measurement: A comparison of electrostatic potentials derived from atomic force microscopy and electrophoretic mobility measurements. Langmuir 2001, 17, 7777-7783.

39. van Zwol, P. J.; Svetovoy, V. B.; Palasantzas, G. Distance upon contact: Determination from roughness profile. Physical Review B 2009, 80.

40.* Czarnecki, J.; Itschenskij, V. Van der Waals attraction energy between unequal rough spherical-particles. Journal of Colloid and Interface Science 1984, 98, 590-591. [Represent together with refs. 47 and 48 the first systematic studies of the effect of roughness on van der Waals forces]

41. Bitter, J. L.; Duncan, G. A.; Beltran-Villegas, D. J.; Fairbrother, D. H.; Bevan, M. A. Anomalous Silica Colloid Stability and Gel Layer Mediated Interactions. Langmuir 2013, 29, 8835-8844.

42. Badaire, S.; Cottin-Bizonne, C.; Stroock, A. D. Experimental Investigation of Selective Colloidal Interactions Controlled by Shape, Surface Roughness, and Steric Layers. Langmuir 2008, 24, 11451-11463.

43. Bevan, M. A.; Prieve, D. C. Direct measurement of retarded van der Waals attraction. Langmuir 1999, 15, 7925-7936.

44. Parsegian, V. A.; Ninham, B. W. Application of Lifshitz theory to calculation of van der Waals forces across thin lipid films. Nature 1969, 224, 1197-1198.

45. Ninham, B. W.; Parsegian, V. A. van der Waals interactions in multilayer systems. Journal of Chemical Physics 1970, 53, 3398-\&.

46. Parsegian, V. A.; Ninham, B. W. Van der Waals forces in many-layered structures generalizations of Lifshitz result for 2 semi-infinite media. Journal of Theoretical Biology 1973, 38, 101-109. 

macroscopic bodies. Physical Review B 1980, 22, 1677-1686. [Represent together with ref. 40 the first systematic studies of the effect of roughness on van der Waals forces]

48.* Mazur, P.; Maradudin, A. A. Effects of surface roughness on the van der Waals force between macroscopic bodies. II. Two rough surfaces. Physical Review B 1981, 23, 695-705. [Represent together with ref. 40 the first systematic studies of the effect of roughness on van der Waals forces]

49. Roach, P.; Shirtcliffe, N. J.; Newton, M. I. Progess in superhydrophobic surface development. Soft Matter 2008, 4, 224-240.

50. Sun, T. L.; Feng, L.; Gao, X. F.; Jiang, L. Bioinspired surfaces with special wettability. Accounts of Chemical Research 2005, 38, 644-652.

51. Zhang, X.; Shi, F.; Niu, J.; Jiang, Y.; Wang, Z. Superhydrophobic surfaces: from structural control to functional application. Journal of Materials Chemistry 2008, 18, 621-633.

52. $\quad$ Cao, L.; Jones, A. K.; Sikka, V. K.; Wu, J.; Gao, D. Anti-Icing Superhydrophobic Coatings. Langmuir 2009, 25, 12444-12448.

53. Choi, C. H.; Kim, C. J. Large slip of aqueous liquid flow over a nanoengineered superhydrophobic surface. Physical Review Letters 2006, 96.

54. $\quad$ Koch, K.; Bhushan, B.; Barthlott, W. Multifunctional surface structures of plants: An inspiration for biomimetics. Progress in Materials Science 2009, 54, 137-178.

55. Yan, Y. Y.; Gao, N.; Barthlott, W. Mimicking natural superhydrophobic surfaces and grasping the wetting process: A review on recent progress in preparing superhydrophobic surfaces. Advances in Colloid and Interface Science 2011, 169, 80-105.

56. Feng, L.; Zhang, Y.; Xi, J.; Zhu, Y.; Wang, N.; Xia, F.; Jiang, L. Petal effect: A superhydrophobic state with high adhesive force. Langmuir 2008, 24, 4114-4119.

57. Liu, K.; Yao, X.; Jiang, L. Recent developments in bio-inspired special wettability. Chemical Society Reviews 2010, 39, 3240-3255.

58. Cassie, A. B. D.; Baxter, S. Wettability of porous surfaces. Transactions of the Faraday Society $1944,40,0546-0550$.

59. Wenzel, R. N. Resistance of solid surfaces to wetting by water. Industrial and Engineering Chemistry 1936, 28, 988-994.

60. Israelachvili, J.; Pashley, R. The hydrophobic interaction is long-range, decaying exponentially with distance. Nature 1982, 300, 341-342.

61. Israelachvili, J. N.; Pashley, R. M. Measurement of the hydrophobic interaction between 2 hydrophobic surfaces in aqueous-electrolyte solutions. Journal of Colloid and Interface Science 1984, 98, 500-514.

62. Christenson, H. K.; Claesson, P. M. Cavitation and the interaction between macroscopic hydrophobic surfaces. Science 1988, 239, 390-392.

63. Christenson, H. K.; Claesson, P. M. Direct measurements of the force between hydrophobic surfaces in water. Advances in Colloid and Interface Science 2001, 91, 391-436.

64. Christenson, H. K.; Claesson, P. M.; Parker, J. L. Hydrophobic attraction - a reexamination of electrolyte effects. Journal of Physical Chemistry 1992, 96, 6725-6728.

65. Ederth, T.; Claesson, P.; Liedberg, B. Self-assembled monolayers of alkanethiolates on thin gold films as substrates for surface force measurements. Long-range hydrophobic interactions and electrostatic double-layer interactions. Langmuir 1998, 14, 4782-4789.

66. Parker, J. L.; Claesson, P. M.; Attard, P. Bubbles, cavities, and the long-ranged attraction between hydrophobic surfaces. Journal of Physical Chemistry 1994, 98, 8468-8480.

67. Carambassis, A.; Jonker, L. C.; Attard, P.; Rutland, M. W. Forces measured between hydrophobic surfaces due to a submicroscopic bridging bubble. Physical Review Letters 1998, 80, 53575360.

68. Ishida, N.; Sakamoto, M.; Miyahara, M.; Higashitani, K. Optical observation of gas bridging between hydrophobic surfaces in water. Journal of Colloid and Interface Science 2002, 253, 112-116. 

temperature dependence of bridging nanobubble induced forces between hydrophobic surfaces. Acs Nano $2008,2,1817-1824$. [First discussion of constant volume approximation for interaction between hydrophobic surfaces]

70. Thormann, E.; Simonsen, A. C.; Hansen, P. L.; Mouritsen, O. G. Interactions between a polystyrene particle and hydrophilic and hydrophobic surfaces in aqueous solutions. Langmuir 2008, 24, 7278-7284.

71. Meyer, E. E.; Rosenberg, K. J.; Israelachvili, J. Recent progress in understanding hydrophobic interactions. Proceedings of the National Academy of Sciences of the United States of America 2006, 103, 15739-15746.

72. Tabor, R. F.; Grieser, F.; Dagastine, R. R.; Chan, D. Y. C. The hydrophobic force: measurements and methods. Physical Chemistry Chemical Physics 2014, 16, 18065-18075.

73. Chandler, D. Interfaces and the driving force of hydrophobic assembly. Nature 2005, 437, 640-647.

74.* $\quad$ Butt, H.-J.; Kappl, M. Normal capillary forces. Advances in Colloid and Interface Science 2009, 146, 48-60. [key paper on capillary forces]

75.* $\quad$ Singh, S.; Houston, J.; van Swol, F.; Brinker, C. J. Superhydrophobicity - Drying transition of confined water. Nature 2006, 442, 526-526. [first direct study of the interaction between superhydrophobic surfaces in water]

76. $\quad$ Brandner, B. D.; Hansson, P. M.; Swerin, A.; Claesson, P. M.; Wahlander, M.; Schoelkopf, J.; Gane, P. A. C. Solvent segregation and capillary evapouration at a superhydrophobic surface investigated by confocal Raman microscopy and force measurements. Soft Matter 2011, 7, 1045-1052.

77.** Wahlander, M.; Hansson-Mille, P. M.; Swerin, A. Superhydrophobicity: Cavity growth and wetting transition. Journal of Colloid and Interface Science 2015, 448, 482-491. [Important systematic study of interactions between superhydrophobic surfaces in water]

78. Hansson, P. M.; Hormozan, Y.; Brandner, B. D.; Linnros, J.; Claesson, P. M.; Swerin, A.; Schoelkopf, J.; Gane, P. A. C.; Thormann, E. Effect of Surface Depressions on Wetting and Interactions between Hydrophobic Pore Array Surfaces. Langmuir 2012, 28, 11121-11130.

79. Hansson, P. M.; Hormozan, Y.; Brandner, B. D.; Linnros, J.; Claesson, P. M.; Swerin, A.; Schoelkopf, J.; Gane, P. A. C.; Thormann, E. Hydrophobic pore array surfaces: Wetting and interaction forces in water/ethanol mixtures. Journal of Colloid and Interface Science 2013, 396, 278-286.

80.** Hansson, P. M.; Swerin, A.; Schoelkopf, J.; Gane, P. A. C.; Thormann, E. Influence of Surface Topography on the Interactions between Nanostructured Hydrophobic Surfaces. Langmuir 2012, 28, 80268034. [first study of interactions between typographical structured hydrophobic surfaces with a systematic variation in surface roughness]

81. Hansson, P. M.; Skedung, L.; Claesson, P. M.; Swerin, A.; Schoelkopf, J.; Gane, P. A. C.; Rutland, M. W.; Thormann, E. Robust Hydrophobic Surfaces Displaying Different Surface Roughness Scales While Maintaining the Same Wettability. Langmuir 2011, 27, 8153-8159.

82. Ensikat, H. J.; Ditsche-Kuru, P.; Neinhuis, C.; Barthlott, W. Superhydrophobicity in perfection: the outstanding properties of the lotus leaf. Beilstein Journal of Nanotechnology 2011, 2, 152-161. 
Recommended:

$17^{* *}$ [systematic comparison between theory and experiment for DLVO forces between rough surfaces] 33* [Interesting discussion about van der Waals forces between rough surface by diffuse layer approch] 40* [Represent together with refs. 47 and 48 the first systematic studies of the effect of roughness on van der Waals forces]

47-48* [Represent together with ref. 40 the first systematic studies of the effect of roughness on van der Waals forces]

69* [First discussion of constant volume approximation for interaction between hydrophobic surfaces]

74* [key paper on capillary forces]

75* [first direct study of the interaction between superhydrophobic surfaces in water]

$77^{* *}$ [Important systematic study of interactions between superhydrophobic surfaces in water]

$80^{* *}$ [first study of interactions between typographical structured hydrophobic surfaces with a systematic variation in surface roughness] 